\title{
GPPS-BJ-2019-0035
}

\section{UNCERTAINTY ANALYSIS OF AERODYNAMIC PERFORMANCE FOR TIP CLEARANCE SIZE OF COMPRESSOR}

\author{
Chi Ma \\ Northwestern Polytechnical University \\ celinema@mail.nwpu.edu.cn \\ Xi'an, Shaanxi, China
}

\author{
Limin Gao \\ Northwestern Polytechnical University \\ gaolm@nwpu.edu.cn \\ Xi'an, Shaanxi, China
}

\author{
Ruiyu Li \\ Northwestern Polytechnical \\ University \\ ryli@mail.nwpu.edu.cn \\ Xi'an, Shaanxi, China
}

\author{
Yu Wu \\ Northwestern Polytechnical \\ University \\ wuyu199589@mail.nwpu.edu.cn \\ Xi'an, Shaanxi, China
}

\author{
Heng Jiang \\ Northwestern Polytechnical \\ University \\ hengjiang@mail.nwpu.edu.cn \\ Xi'an, Shaanxi, China
}

\begin{abstract}
Blade tip clearance plays a vital role on aerodynamic performance of compressor. Slightly change of tip clearance size can result in violent change of the tip clearance flow field, and subsequently seriously affect the aerodynamic performances. Since the clearance size is subject to variabilities influenced by working conditions, manufacture, complex service process and some other factors, to improve the aerodynamic performance of tip clearance region, it is essential to study the influence of the tip clearance uncertainty on the flow field and aerodynamic performance parameters. In this paper, the tip clearance size distributions over different rotating speeds are respectively mapped. Generally, the tip clearance sizes for different working conditions satisfy the Gaussian distributions, where the mean value and standard deviation can be changed according to the conditions. NonIntrusive Polynomial Chaos (NIPC) is introduced here to achieve the uncertainty quantification (UQ) of output aerodynamic performance parameters of compressor. The various impacts of input tip clearance size uncertainty under different working conditions are investigated here, as well as the differences and relations of these impacts on the aerodynamic Quantity of Interest (QoI). With this methodology, it is practicable to analyse the specific effects of stochastic tip clearance on compressor aerodynamic performance for different working conditions. The UQ results show that part rotating speeds conditions are more sensitive to the tip clearance size uncertainty, especially the near stall operating points. The results of this research provide some useful information to improve compressor design process and some meaningful hints for further robust design. The results
\end{abstract}

shown in this paper can serve as study case for further comparisons with other UQ methodology and results as well.

\section{INTRODUCTION}

The characteristics of tip clearance are small size, many influencing factors, and locating in a complex flow structure. What's more, the effect of these changes always enlarges in real compressor operation, which make it a mainly concern parameter in compressor, especially the high rotating speed ones.

Tip clearance size of compressor is a geometric small but very sensitive parameter, which can drastically affect the loss, performances, the unsteady flow behaviors and aerodynamic stability (Mao et al., 2017; Seshadri et al., 2014; Xiang et al., 2018) and it has significant changes in different operating points which may results in the damage of the whole engine and therefore also plays an important role on the engine safety.

The tip clearance size uncertainty can cause by many factors such as temperature difference among casing, blades and disk; thermal loads distributions; operating conditions; tolerance difference of casing and blades; concentricity error of two-spool rotor; coating thickness of casing surface and so on (Hirsch et al., 2016).

The complex flow structure of transonic rotor tip clearance region has been widely discussed to better understand the flow mechanism, which can enlarge the effect of tip clearance size. Generally, the flow field is affected by shock wave, tip leakage vortex, pressure difference between the pressure side and the suction side. Specifically, shockvortex can induce the periodic breakdown of tip leakage vortex at near stall condition as presented in the work of Yanfeng Zhang et al. (Zhang et al., 2008); Axially non- 
uniform tip clearances in some operating conditions can form beneficial leakage flow, which is good for reducing the flow blockage in passage by blowing down the low-energy fluids at tip region (Ma et al., 2013). In addition, the research of Jie Gao et al. (Gao et al., 2014) found that tip clearance size can directly change the leakage vortex: leakage vortex did not form in small size while in appropriate size, it was possible to see the leakage vortex breakdown.

In recent years, the importance of tip clearance uncertainty has been stressed by many researchers. Zhiyi Liu (Liu et al., 2013) set four schemes of tip clearance height uncertainty using non-intrusive probabilistic collocation to investigate its effect on the aerodynamic performance of Rotor 37. TN Satish (Satish et al., 2014) measured the tip clearances effect of fan rotor under dynamic rotating condition and paid a considerable attention on the uncertainty of measurement system. Pranay Seshadri (Seshadri et al., 2014) studied the influence of tip clearance variation in aerodynamic performances and showed that higher efficiency in tip region can produce higher mixed-out losses. Pranay Seshadri (Seshadri et al., 2015) concentrated on the uncertainty of leakage mass flow, leakage whirl velocity, and radial flow angle, and stressed on the influence of leakage flow on the hub pressure deficit at both on- and off-design conditions. Remy Nigro (Nigro et al, 2017) studied correlated uncertainties on the blade geometry of the NASA Rotor 37. Dirk Wunsch (Wunsch et al, 2015) cooperated with Remy Nigro in developing a probabilistic collocation method in combination with a sparse grid approach for studying simultaneously the combined effect of operational (total inlet pressure, static outlet pressure) and geometrical (tip clearance, leading edge angle and trailing edge angle) input uncertainties on output uncertainties. In addition, skewness and kurtosis are introduced in this work to help reconstruct the output probability density functions. Cosimo Maria Mazzoni et al. (Mazzoni et al, 2018) quantified the impact of the random variation of clearance size in a multistage steam turbine combining unsteady computational fluid dynamics (CFD) and nonintrusive uncertainty quantification (UQ) simulation. They showed that the pressure coefficient distribution at the exit is strongly affected by tip clearance uncertainty.

Most of the studies about tip clearance uncertainty are based on the tip clearance size variation over different operating points. And some researches providing tip clearance size distribution are based on hypothetical distribution form or assuming that the tip clearance variation for certain working points equals to some fixed percent of nominal tip clearance size. However, under real working circumstance, the statistical analysis of experimental measured data shows a nonlinear relation between tip clearance size and corresponding variations at different partial rotating speeds.

Therefore, this work intents to quantify the input tip clearance uncertainty effect for different partial rotating speeds on QoI, including loss, efficiency and mass flow rate. In addition, some detail information such as leakage mass flow variations and the uncertainty propagation along the chordwise also discussed. Those results will helpful to make sure the sensitivity region in the passage which will helpful to improve robust aerodynamic blade design.

\section{TIP CLEARANCE SIZE DISTRIBUTION CHARACTERISTICS AND RESEARCH OBJECT}

\section{Distribution characteristics of tip clearance size}

Component test of a titanium alloy transonic compressor stage was experimentally carried out to measure the tip clearance sizes by capacitance sensor through the full operating rotating range to find out its change tendency and amplitude. The nominal design speed is $12000 \mathrm{rpm}$, and the tip speed is higher than $400 \mathrm{~m} / \mathrm{s}$. That nominal operational condition is like the one used on the NASA Rotor37. Figure 1 provides the tip clearance size measured in the steady state conditions for various rotating speeds. And all the mean value $\mu$ in Figure 1 are all normalized with the mean value of experimental data at $100 \%$ speed, and all the standard deviation $\sigma$ of tip clearance size are all normalized with the corresponding $\mu$ of the same part rotating speed. It's intuitive to magnify 100 times all the standard deviation data to identify the difference for various rotating speed. For the data measurement at each operating speed, more than 200 samples were recorded to assure the richness of sample space. Kolmogorov Smirnov test (K-S test) was introduced to obtain the probability distribution function (PDF) of the tip clearance samples. The experimental results show that the tip clearance size variability at various operating points are all satisfied Gaussian distributions, which can be written as $\left(\mu, \sigma^{2}\right)$. This very probability distribution form is the key point for quantifying the effect of tip clearance size uncertainty in this work.

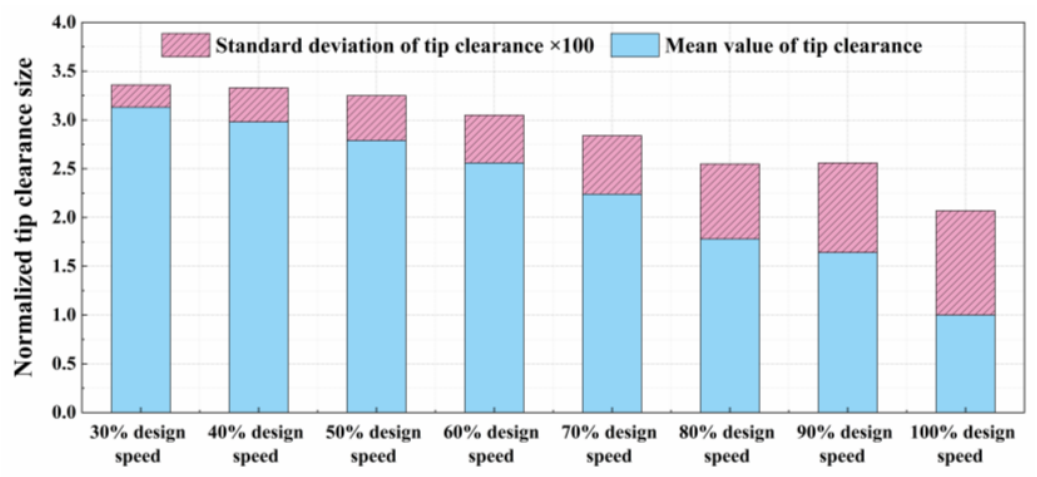

Figure 1 Normalized measurement data of rotor blade tip clearance for different operating points 
From Figure 1, it can be concluded that the mean value of tip clearance size becomes smaller when the rotating speed grows without exception. For the normalized standard deviation value, it becomes larger when the rotating speed grow. This trend is commonly suitable at all the speed conditions. However, one can highlight that the relationship between the standard deviation and the corresponding mean value of tip clearance size is not linear, which means they do not strictly follow a clear mathematical relationship or a certain percent of mean value of tip clearance. For a more practical research, it is better to follow a series of experimental measurement data like showed in this paper.

\section{Research object and validation}

As mentioned above, Rotor 37 was chosen to help studying the tip clearance uncertainty in this work. The design speed is $17188 \mathrm{rpm}$ where the design total pressure ratio is 2.106 and the design flow mass rate is $2.188 \mathrm{~kg} / \mathrm{s}$. Ahead of that research based on experimental data, a CFD study case on the Rotor 37 was carried out.

According to the experimental results presented in (Chan, 1996), efficiency and total pressure ratio in the spanwise direction simulated at $98 \%$ of the choked flow are compared with the experimental data to validate the simulation settings (see Figure 2). The cell width of y along the blade wall was set to $0.001 \mathrm{~mm}$ ensuring that the corresponding $\mathrm{y}+$ is smaller than six. The Spalart-Allmaras (S-A) turbulence model (Liu et al. 2014) is used as closure model ( $y+$ is limited to less than ten). For the QoI, it demonstrated a good agreement in trends between CFD simulations and experimental data. And the relative Mach number contour at $95 \%$ span in $98 \%$ choked mass flow condition obtained by CFD also agree well with experimental results, as shown in Figure 3.

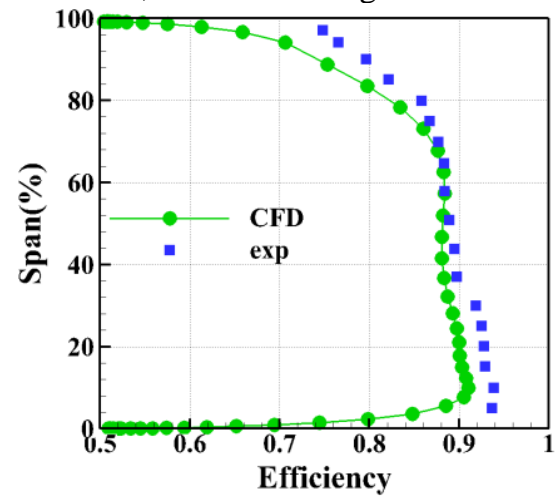

(a) Efficiency

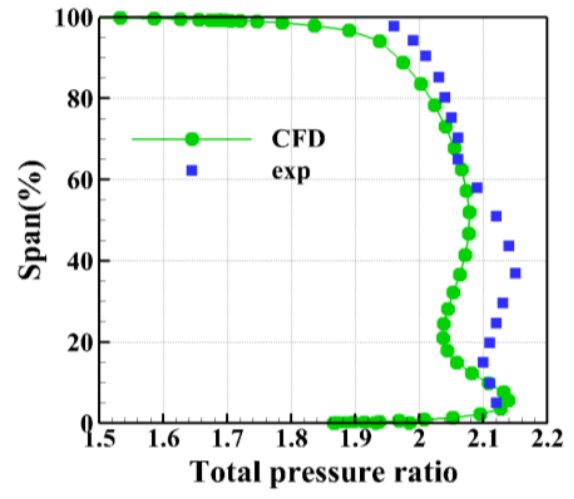

(b) Total pressure ratio

Figure 2 Spanwise distribution comparison of CFD results and experimental results (Chan et al., 1996) at $98 \%$ choked flow

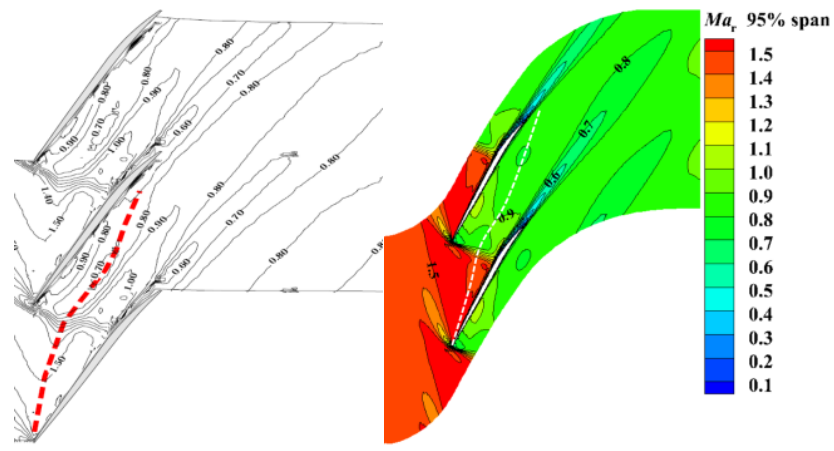

(a) Experiment (Suder, 1998)

(b) CFD result

\section{Figure 3 Relative Mach number distribution at $98 \%$} choked mass flow

\section{UNCERTAINTY QUANTIFICATION METHOD}

The NIPC belongs to stochastic spectral method and has gradually caught the attention due to its advantages in prediction accuracy and the effectiveness. This method is widely used for UQ. For CFD simulation, NIPC can tremendously reduce the number of simulations needed to predict the stochastic output moments (Montomoli et al., 2015) and can regard the CFD solving package as a black box. That NIPC methodology was used in this work to perform a UQ of the aerodynamic output performances of interest based on the input tip clearance variability.

The main theoretical basis and solving principle of the NIPC can be found in the reference (Eldred et al., 2009). For NIPC method, which can decompose a stochastic output $(Y)$ into a series stochastic orthogonal polynomial $\psi_{i}(\xi)$ with deterministic coefficients $a_{j}$.

$$
\begin{aligned}
& Y=\sum_{j=0}^{Q-1} a_{j} \psi_{i}(\xi) \\
& Q=\frac{(p+n) !}{p ! n !}
\end{aligned}
$$

Where, $n$ is the number of random variables, $Q$ is the number of terms in NIPC. The $p$ is the order of NIPC, which depends on the accuracy requirement in different cases. $\psi_{j}$ is $j$ order Hermite polynomial of random variables (Ouyang, 2009), and $a_{j}$ is coefficient of NIPC.

The mean value $\left(\mu\left(Y\left(x_{m_{1}}, x_{m_{2}}, \cdots, x_{m_{n}}\right)\right)\right)$ and variance $\left(\sigma^{2}\left(Y\left(x_{m_{1}}, x_{m_{2}}, \cdots, x_{m_{n}}\right)\right)\right)$ of the random variable response can be obtained by (3) and (4):

$$
\begin{aligned}
\mu\left(Y\left(x_{m_{1}}, x_{m_{2}}, \cdots, x_{m_{n}}\right)\right) & =a_{0} \\
\sigma^{2}\left(Y\left(x_{m_{1}}, x_{m_{2}}, \cdots, x_{m_{n}}\right)\right) & =\sum_{j=1}^{Q-1}\left[a_{j}^{2}\left\langle\psi_{j}^{2}\right\rangle\right]
\end{aligned}
$$


Where, $Y\left(x_{m_{1}}, x_{m_{2}}, \cdots, x_{m_{u}}\right)$ presents the output of a system, and $x_{m_{i}}(i=1, \ldots, n)$ are the uncertainty variables, In this paper, $Y\left(x_{m_{1}}, x_{m_{2}}, \cdots, x_{m_{n}}\right)$ could be the value of aerodynamic performance parameters (total pressure ratio, mass flow rate, isotropic efficiency and so on) and $x_{m_{i}}$ is uncertain tip clearance size value at the Gaussian Hermite integrand points of the PDF. For the above two formulas it can be seen that the mean value $\left(\mu\left(Y\left(x_{m_{1}}, x_{m_{2}}, \cdots, x_{m_{n}}\right)\right)\right)$ is 0 order term of the coefficient of the polynomial chaos expansion, and the variance $\left(\sigma^{2}\left(Y\left(x_{m_{1}}, x_{m_{2}}, \cdots, x_{m_{n}}\right)\right)\right)$ is the quadratic sum of the other coefficients. By solving polynomial coefficients, it can be convenient to calculate the responded statistical properties. So, the core of this method is the way to get the solution of the polynomial coefficients.

The coefficients of each terms can be represented as following:

$$
a_{j}=\sum_{m_{1}=1}^{m} \cdots \sum_{m_{n}=1}^{m} Y\left(x_{m_{1}}, x_{m_{2}}, \cdots, x_{m_{n}}\right) \frac{\psi_{j}\left(x_{m_{1}}, x_{m_{2}}, \cdots, x_{m_{n}}\right)}{\left\langle\psi_{j} \psi_{j}\right\rangle} \prod_{k=1}^{n} w_{m_{k}}
$$

Where, $m$ is the number of integrand collocation points according to $p . w_{m_{k}}(k=1,2, \cdots, m)$ is weight, which is constant and can be found in (Ouyang, 2009).

On the NASA Rotor 37, as presented in the literature (Mazzoni et al., 2018; Ghisu et al., 2018), $4^{\text {th }}$ order NIPC (five integrand collocation points) is chosen in this paper to quantify the output variability of the aerodynamic performances due to the of tip clearance uncertainty.

\section{RESULTS AND DISCUSSION}

\section{Operating points selection}

To limit the size of this paper, $100 \%$ design speed, $90 \%$ design speed and $70 \%$ design speed are chosen as operating conditions for a detailed discussion.

Figure 4 shows the total pressure ratio and isentropic efficiency at three speeds to find out how the input tip clearance uncertainty affects the output aerodynamic performances at various speeds. For these rotational speeds, three typical operating points (the near stall, the peak efficiency and the blockage conditions) are chosen to quantify the uncertainties of the QoI corresponding to the mass flow rate, the total pressure ratio and the isentropic efficiency in each speed. The selected conditions are marked with a yellow marker and a tiny arrow.

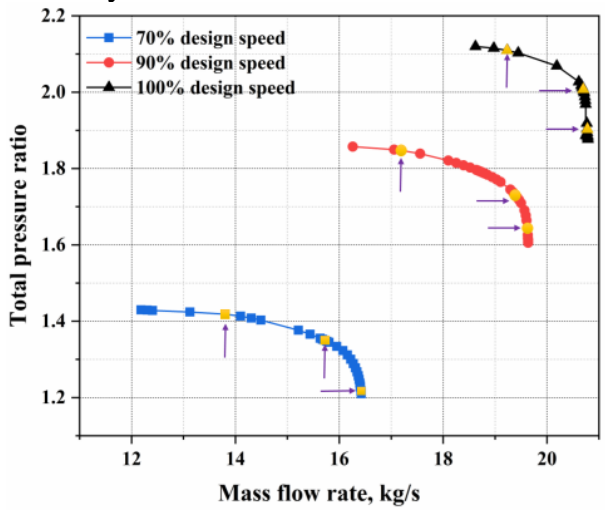

(a) Total pressure ratio

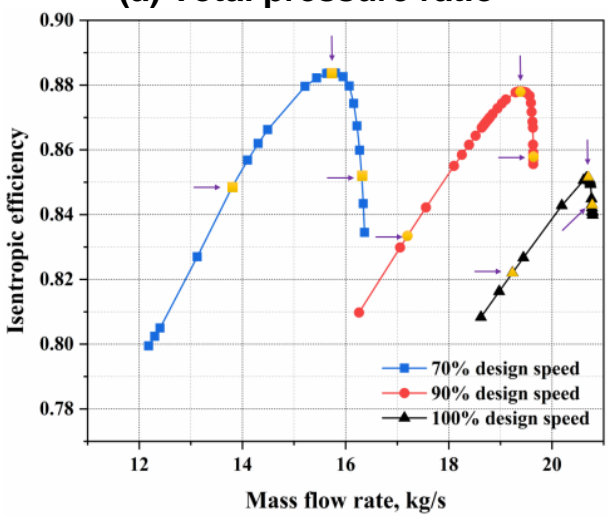

(b) Isentropic efficiency

\section{Figure 4 Performance parameters comparison at different speeds}

\section{Effects on aerodynamic performances}

Mean value and standard deviation of mass flow rate $\left(m_{\mathrm{a}}\right)$, total pressure ratio $\left(\pi_{\mathrm{t}}\right)$ and isentropic efficiency $\left(\eta_{\mathrm{is}}\right)$ for three speeds at blockage, peak efficiency and near stall conditions are illustrated in Figure 5-7. All the mean values shown in these figures are real values, but the standard deviation $(\sigma)$ are normalized with corresponding mean value. So that we can clearly know the fluctuation amplitude differences with respect to the corresponding mean value among different conditions.

From these three figures, all the mean values about different aerodynamic performance parameters and their trends are all similar to the results of deterministic analysis method. In Figure 5, for $100 \%$ design speed conditions, the mass flow rate is higher than the other two speeds in three type (blockage, peak efficiency and near stall) operating points. And the trend of total pressure ratio in Figure 6 has the same trend as mass flow rate changes. However, there is something different appeared in Figure 7. There is no doubt that the peak efficiency conditions have the highest isentropic efficiency in three speeds. But at peak efficiency operating point, the highest isentropic efficiency is in $70 \%$ design speed condition.

In this section, the main idea is to know how the tip clearance size uncertainty affect the overall aerodynamic performance parameters, so the standard deviation changes of QoI are the most concerned. The descending order of tip clearance size fluctuation amplitude (the standard deviation in Figure 1) is $100 \%$ design speed, $90 \%$ design speed and $70 \%$ design speed. If the QoI variation trend are not consistent with the tip clearance size fluctuation, the sensitivity relationship between different operating conditions can be distinguished. 

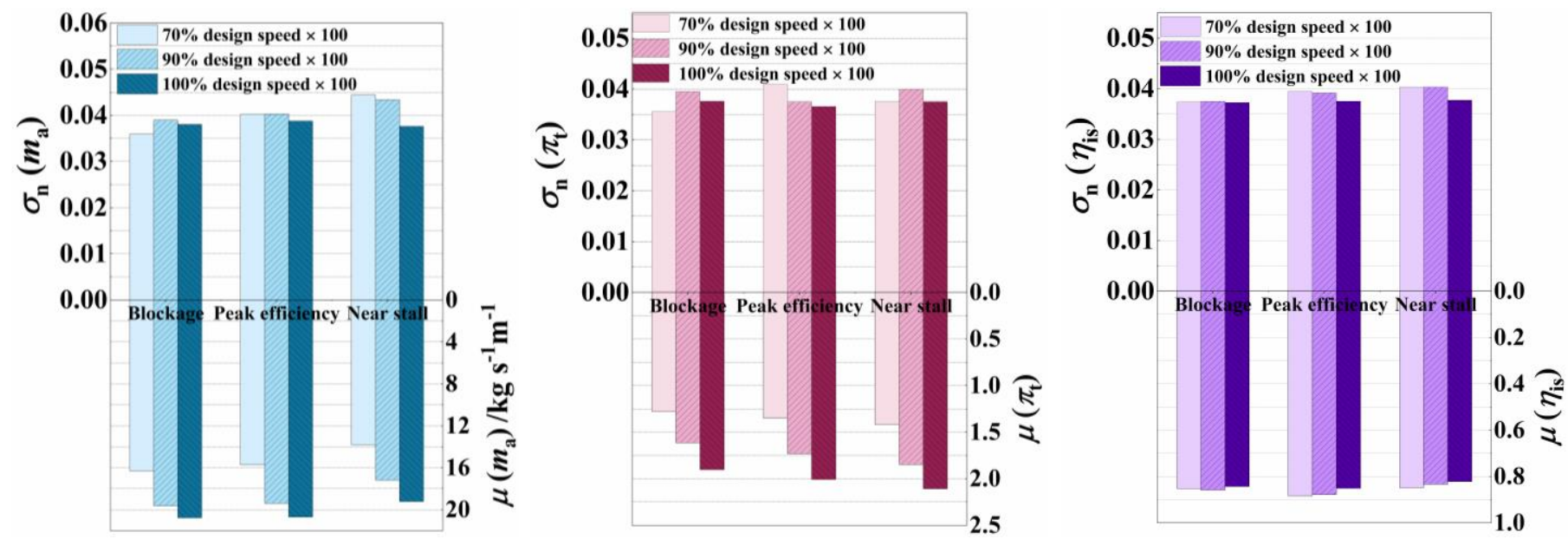
Figure 5 Mean value and normalized Figure 6 Mean value and normalized Figure 7 Mean value and normalized standard deviation of mass flow standard deviation of total pressure rate for different speeds ratio for different speeds

\section{standard deviation of isentropic efficiency for different speeds}

The mass flow rate changes with the tip clearance uncertainty are shown in Figure 5. For the nominal design speed, the most sensitive operating point is peak efficiency while the least sensitive operating point is near stall. And the difference among these three types of operating points is slight, in which the range of variations is $0.00116 \%$. For the part speed conditions, the near stall operating condition is the most sensitive, where the biggest fluctuation amplitude is $0.04441 \%$ for $70 \%$ design speed. On the other hand, the blockage operating condition is least sensitive, where the smallest fluctuation amplitude is $0.03592 \%$ for $70 \%$ design speed. The range of variations is $0.00848 \%(70 \%$ design speed) and $0.00442 \%$ (90\% design speed).

The fluctuation amplitude of tip clearance size for $70 \%$ design speed is medium, but the mass flow fluctuation amplitude for near stall operating point at $70 \%$ design speed is most affected by this uncertainty. While the effect of tip clearance uncertainty on the mass flow rate of $100 \%$ design speed condition is slightly.

The total pressure ratio changes with the tip clearance uncertainty are shown in Figure 6. For the $100 \%$ nominal design speed, the most sensitive operating point is blockage operating point while the least sensitive operating point is the peak efficiency. The range of variations of $100 \%$ design speed is $0.00104 \%$. For the part speed conditions, the most sensitive operating point is the peak efficiency for $70 \%$ design speed, while the least sensitive one is the blockage for $70 \%$ design speed. For this parameter, there is no clear operating type which is always sensitive to the tip clearance uncertainty among these three type operating points. The range of variations of $\pi_{\mathrm{t}}$ are $0.00539 \%$ (70\% design speed) and $0.00249 \%$ ( $90 \%$ design speed).

The total pressure ratio of $70 \%$ design speed is the most sensitive condition affected by tip clearance uncertainty while the $\pi_{\mathrm{t}}$ of $100 \%$ design speed does not be affected by the uncertainty. These result about $\pi_{\mathrm{t}}$ is the same as $m_{\mathrm{a}}$ 's discussed above.

The isentropic efficiency changes with the tip clearance uncertainty are shown in Figure 7. For the $100 \%$ design speed, the fluctuation amplitude in three operating points are almost the same, the range of variations is just $0.00046 \%$. So $\eta_{\text {is }}$ shows good robustness on the tip clearance size uncertainty for this rotating speed. For the part speed conditions, the most sensitive operating point occurs at near stall for $90 \%$ design speed while the least sensitive one is the blockage for $70 \%$ design speed. The near stall operating points for all these three rotating speeds reflect the biggest fluctuation, which means the near stall operating points, relatively speaking, is sensitive to tip clearance size uncertainty. The range of variations of $\eta_{\text {is }}$ are $0.00297 \%$ (70\% design speed) and $0.00284 \%$ (90\% design speed). The fluctuation of $\eta_{\text {is }}$ is slightly smaller at the various speeds unlike the other two aerodynamic performance parameters.

\section{Effects on leakage mass flow}

Tip clearance size is closely associated with tip leakage mass, which strongly affect the tip clearance flow field. The definition of leakage flow mass is following:

$$
m_{\text {leakage }}=\int_{S} \rho \cdot \vec{w} \cdot d S
$$

The leakage surface $(S)$ used in the Eq. (6) is shown in Figure 8 . It is located in the tip clearance domain and vertical to the tip of blade. This surface is cut into 30 parts and numbered, preparing for study the leakage mass flow changes along the chord in the following section.

Figure 9 shows the tip clearance leakage mass flow distribution along the chord superimposed standard deviation to find out which part contributes the most leakage mass flow. And Figure 10 compares the fluctuation amplitude among three operating points for each speed.

For the mean value of the $m_{\text {leakage }}$ for three rotating speeds, the front part and rearing part are two regions where $m_{\text {leakage }}$ are relatively higher than other parts, this phenomenon is associated with the leakage vortex and inducing double leakage vortex. However, in the near stall operating point for $90 \%$ and $100 \%$ rotating speeds the rear high $m_{\text {leakage }}$ moves to middle part. As the rotating speed goes down, the $m_{\text {leakage }}$ 
tendency for three types operating points along the chord begins to converge.

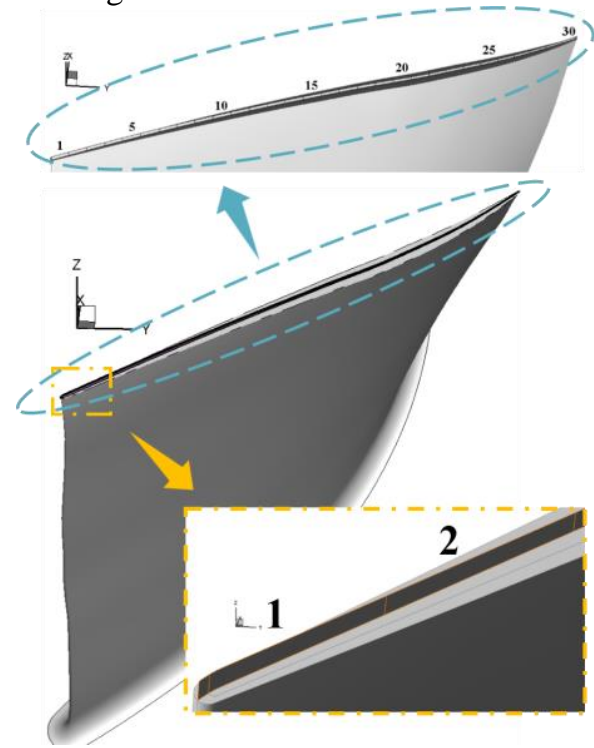

Figure 8 Leakage mass flow surface
This work is more concerned with $m_{\text {leakage }}$ fluctuation caused $\left(\sigma_{\text {leakage }}\right)$ by tip clearance size uncertainty shown in Figure 10 . For $100 \%$ speed condition, the high fluctuation location is at about the front part of the chord range from $6.7 \%$ $(2 / 30)$ to $12 \%(6 / 30)$, and the other high fluctuation location is at the middle part of the chord range from $56.7 \%(17 / 30)$ to $73.3 \%$ (22/30); For $90 \%$ speed condition, the high fluctuation location is at about the front part of the chord is about $13.3 \%$ (4/30), and the other high fluctuation location is at the middle part of the chord range from $50 \%(15 / 30)$ to $83.3 \%(25 / 30)$; For $70 \%$ speed condition, the high fluctuation location is at about the front part of the chord range from $16.7 \%(5 / 30)$ to $30 \%(9 / 30)$, and the other high fluctuation location is at the rear part of the chord range from $93.3 \%$ (28/30) to $100 \%$ (30/30). In summary, with the fluctuation amplitude of tip clearance size goes up, the high leakage mass flow fluctuation location moves forward.

Comparing the mean value of $m_{\text {leakage }}$ and $\sigma_{\text {leakage }}$ for certain rotating speed, the tendency between these two parameters are relevant but are not an exact match, which reflects the necessity of uncertainty analysis of complex flow field.

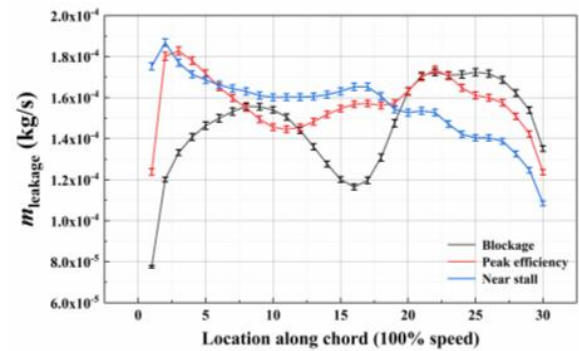

(a) $100 \%$ speed

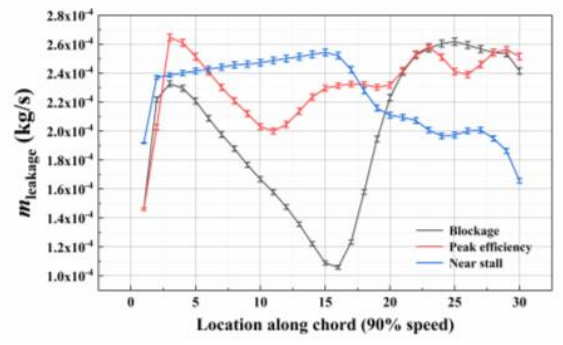

(b) $90 \%$ speed

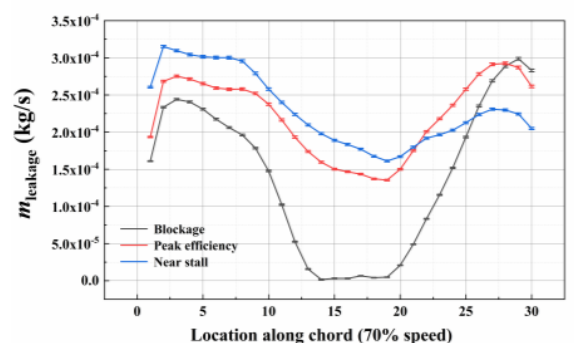

(c) $70 \%$ speed

Figure 9 Leakage along chord distribution

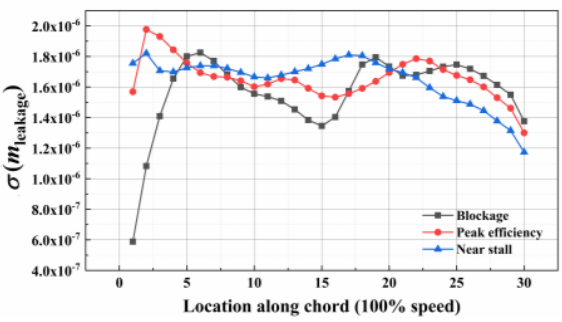

(a) $100 \%$ speed

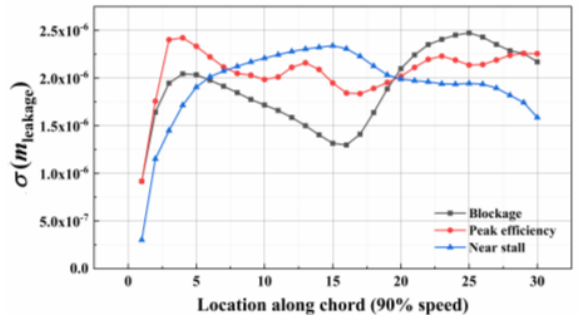

(b) $90 \%$ speed

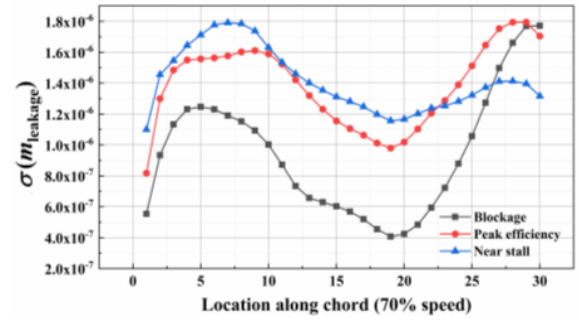

(c) $70 \%$ speed

Figure 10 Standard deviation of relative leakage distribution along chord

\section{Effects on tip clearance flow field}

Based on the above discussions, it can be concluded that the $70 \%$ rotating speed at near stall operating point is sensitive. To investigate the flow mechanism of QoI fluctuation caused by tip clearance uncertainty, statistical characteristics of static pressure coefficient $\left(C_{\mathrm{p}}\right)$ distribution at near stall operating point of $70 \%$ speed are shown in Figure 11. As shown in Figure 11 (a), most blade loading is located in the first $20 \%$ chord length while the loading near the traling is little.

The static pressure coefficient is defined as follows:

$$
C_{\mathrm{p}}=\left(P-P_{\text {in }}\right) /\left(P_{\text {in }}^{*}-P_{\text {in }}\right)
$$

Where, $P$ and $P_{\text {in }}$ denote the local and inlet static pressure, $P^{*}{ }_{\text {in }}$ denote the total pressure at inlet.

Figure 11(b) displays the standard deviation of $C_{\mathrm{p}}$. It can be observed that the maximum $C_{\mathrm{p}}$ on the pressure surface (PS) and the first peak $C_{\mathrm{p}}$ on the suction surface (SS) are in the same normalized axial chord location (about 0.05). Comparing this location with Figure 9 (c) and Figure 10 10(c) (in the middle of part 1 part 2), it is also the place that the tip leakage mass flow begins. Therefore, it can be concluded that these two peak $C_{\mathrm{p}}$ is caused by the leakage flow fluctuation induced initially from tip clearance uncertainty. The $2^{\text {nd }}$ peak $C_{\mathrm{p}}$ on SS 
is consistent with the maximum relative Mach number (Ma_relative) as shown in Figure 12(a). The tip clearance uncertainty disturbs the tip leakage vortex track and consequently affect the blockage region, the blockage is the core reason dominate the fluctuation in the flow field. What's more, its interference with shock wave affects the acceleration on the SS. The statistical characteristics of $M a \_$relative can be seen in Figure 12. The biggest fluctuation region illustrated Figure 12(b) also proved that the blockage uncertainty caused by tip clearance is the critical factor.

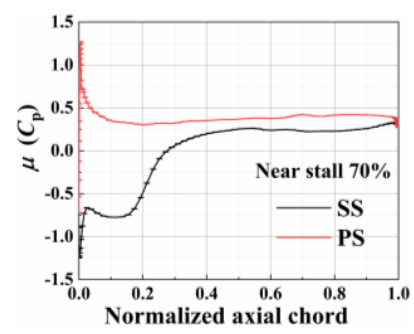

(a) mean value

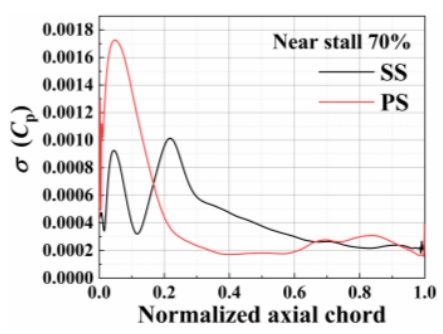

(b) Standard deviation
Figure 11 Static pressure coefficient of the blade tip at $98 \%$ span at near stall condition of $70 \%$ speed $\mu($ Ma_relative $)$

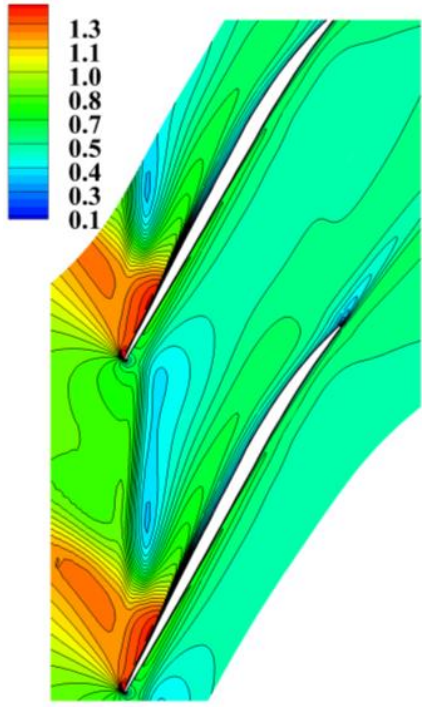

(a) mean value $\sigma\left(M a \_\right.$relative $)$

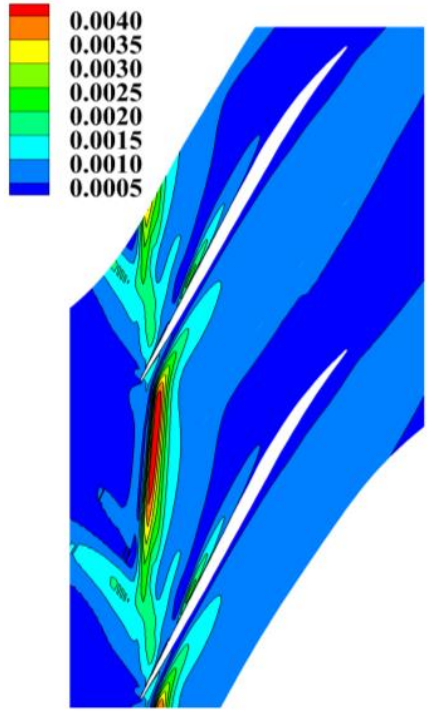

(b) Standard deviation
Figure 12 Relative Mach number contour at $98 \%$ span at near stall condition of $70 \%$ speed

\section{CONCLUSIONS}

A UQ analysis using a NIPC methodology coupled with CFD simulations has been applied to analyses the effect of tip clearance size uncertainties characterized by a Gaussian PDF based on experimental measuring data. The effect of the input uncertainty of tip clearance size on the uncertainty of QoI have been drawn according to the near stall, peak efficiency and blockage conditions for three rotating speeds. It can be concluded that:

(1) Analysis of experimental data shows that the uncertainty for the various rotating speeds was nonlinear with respect to the evolution of the mean value of the tip clearance size. When the rotating speed become higher, the normalized fluctuation amplitude of tip clearance increases.

(2) Among three QoIs, the most affected parameter is $m_{\mathrm{a}}$ while the least affected parameter is $\eta_{\text {is }}$; the most sensitive rotating speed occur in $70 \%$ rotating speed while the $100 \%$ nominal design speed is the least sensitive condition; and the near stall operating point is the most sensitive type operating conditions among these three types.

(3) The most sensitive region caused by tip clearance size uncertainty is the blockage. The tip clearances uncertainty results in tip clearance leakage vortex track fluctuation, and concequently change the blockage in the flow field.

\section{NOMENCLATURE}

\section{Symbols \\ $\mu=$ Mean value \\ $\sigma=$ Standard deviation \\ $\pi=$ Pressure ratio \\ $m=$ Mass flow rate \\ $\eta=$ Efficiency \\ $w=$ Relative velocity}

\section{ACKNOWLEDGMENTS}

The authors would like to express appreciation for the support of the National Natural Science Foundation of China (NSFC) under the Grant No. 51790512; the Overseas Expertise Introduction Project for Discipline Innovation (111 Project) under Grant No. B17037; and Industry-UniversityResearch Cooperation Project of Aero Engine Corporation of China (AECC) under Grant No. HFZL2018CXY011-1 and MIIC.

\section{REFERENCES}

[1] Mao X. C., Liu B. (2017). Numerical investigation of tip clearance size effect on the performance and tip leakage flow in a dual-stage counter-rotating axial compressor. IMeche, Journal of Aerospace Engineering, 231(3), 474-484. doi:10.1177/0954410016638878

[2] Seshadri P., Shahpar S., Geoffrey T. (2014). Parks. Robust compressor blades for desensitizing operational tip clearance variations. Proceedings of ASME Turbo Expo 2014: Turbine Technical Conference and Exposition. June 16 - 20, Düsseldorf, Germany. P. 1-12. doi:10.1115/gt2014-26624

[3] Xiang H. H., Ning G., Gao J., et al. (2018). Effect of circumferential non-uniform tip clearance on performance of axial compressor. Acta Aeronautica Et Astronautica Sinica, 39(2), 121491 (in Chinese).

[4] Hirsch C., Wunsch D., Szumbarski J., et al. (2016). Uncertainty management for robust industrial design in aerodynautics. Switzerland: Springer.

[5] Zhang Y. F., Chu W. L., Lu X.G. (2008). Numerical simulation of the flow characteristic of tip leakage flow in a transonic axial-flow compressor at near stall condition. Journal of Aerospace Power, 23(7), 1293-1298. (in Chinese). [6] Ma H., Wang Z., Zhang J. (2013). Experimental study of effects of axially non-uniform tip clearances on the aerodynamic performance of a compressor cascade. Aiaa 
Aerospace Sciences Meeting Including the New Horizons Forum and Aerospace Exposition. doi:10.2514/6.2009-1243

[7] Gao J., Zheng Q., Xu T. B., et al. (2014). Effect of tip leakage vortex breakdown on losses in turbines. Acta Aeronautica Et Astronautica Sinica, 35(5), 1257-1264. (in Chinese)

[8] Liu Z. Y., Wang X. D., Kang S. (2013). Non-deterministic CFD simulations on the effect of uncertain tip clearance on an axial rotor performance. Advanced Materials Research, 860863(2014), 1499-1505. doi: 10.4028/www.scientific.net/amr.860-863.1499

[9] Satish T. N., Murthy R., Singh A. K. (2014). Analysis of uncertainties in measurement of rotor blade tip clearance in gas turbine engine under dynamic conditions. IMeche, Journal of Aerospace Engineering, 228(5), 652-670. doi:10.1177/0954410013478523

[10] Seshadri P., Parks G. T., Shahpar S. (2015). Leakage Uncertainties in Compressors: The Case of Rotor 37. Journal of Propulsion \& Power, 31(1). doi:10.2514/1.b35039

[11] Nigro R., Coussement G., Wunsch D., et al. (2017). Uncertainty Quantification in Internal Flows Applied to Manufacturing Tolerances. 55th AIAA Aerospace Sciences Meeting, 9 - 13 January Grapevine, Texas. doi:10.2514/6.2017-1200

[12] Wunsch D., Hirsch C., and Nigro R. (2015). Quantification of combined operational and geometrOical uncertainties in turbo-machinery design. Proceedings of ASME Turbo Expo 2015: Turbine Technical Conference and Exposition, June 15 - 19, Montréal, Canada, 1-11. doi: $10.1115 /$ gt2015-43399.

[13] Mazzoni C. M., Ahlfeld R., Rosic B., et al. (2018). Uncertainty quantification of leakages in a multistage simulation and comparison with experiments. Journal of Fluids Engineering, 140(2), 021110. doi:10.1115/1.4037983

[14] Liu Z. Y., Wang X. D., and S Kang. (2014). Nondeterministic cfd simulations on the effect of uncertain tip clearance on an axial rotor performance. Advanced Materials Research, 860-863:1499-1505. doi:10.4028/www.scientific.net/amr.860-863.1499

[15] Chan C. N. (1996). Validation of CFD codes for propulsion system components. Journal of Materials Science Materials in Medicine, 22(9):2035-2044.

[16] Suder K. L. (1998). Blockage Development in a Transonic, Axial compressor Rotor. Journal of Turbomachinery, 120(3), 465-476. doi:10.1115/1.2841741

[17] Montomoli F., Carnevale M. and D'Ammaro A. (2015). Uncertainty quantification in computational fluid dynamics and aircraft engines. Heidelberg: Springer International Publishing.

[18] Eldred M. S. and Burkard J. (2009). Comparison of nonintrusive polynomial chaos and stochastic collocation methods for uncertainty quantification. 47th AIAA Aerospace Sciences Meeting Including the New Horizons Forum and Aerospace Exposition; Jan 5-8; Orlando, Florida. p. 1-20. doi: 10.2514/6.2009-976

[19] Ouyang J. (2009). Numerical analysis. Beijing: Higher Education Press. (in Chinese).

[20] Ghisu T. and Shahpar S. (2018). Affordable uncertainty quantification for industrial problems: application to aero- engine fans. Journal of Turbomachinery. 140(6). doi:10.1115/1.4038982 